\title{
Rapid changes in capillary morphology and architecture in a patient with dermatomyositis
}

\author{
Sung-Jo Park ${ }^{1}$, In-Ho Choi ${ }^{2}$, and Hyun-Sook Kim ${ }^{1}$
}

Departments of ${ }^{1}$ Internal Medicine and ${ }^{2}$ Pathology, Soonchunhyang University Seoul Hospital, Seoul, Korea

Received: October 9, 2017

Revised : October 10, 2017

Accepted: October 10, 2017

\section{Correspondence to}

Hyun-Sook Kim, M.D.

Tel: +82-2-710-3214

Fax: +82-2-709-9554

E-mail : healthyra@schmc.ac.kr
Dermatomyositis (DM) is an idiopathic inflammatory myopathy with characteristic cutaneous findings, frequently accompanied by proximal muscle weakness. Microvascular involvement is a remarkable characteristic of DM; the observed nailfold capillaroscopic (NFC) changes exhibit a scleroderma-like pattern and may be diagnostically useful.

A 75-year-old male was referred with proximal arm swelling and a facial rash. He had been treated for hypertension, diabetes mellitus, gout, and had undergone a low anterior resection to treat rectal cancer 12 years prior. Physical examination revealed painful edema of both upper extremities and a widespread facial rash. Laboratory tests revealed an elevated erythrocyte sedimentation rate of 103 $\mathrm{mm} / \mathrm{hr}$, and a C-reactive protein level of $1.15 \mathrm{mg} / \mathrm{dL}$. The lactate dehydroge- nase level was $253 \mathrm{U} / \mathrm{L}$ (normal range, o to 250); that of aspartate transaminase was $52 \mathrm{U} / \mathrm{L}$ (range, o to 40), that of alanine transaminase was $27 \mathrm{U} / \mathrm{L}$ (normal range, $O$ to 41 ), that of creatine kinase was $354 \mathrm{U} / \mathrm{L}$ (normal range, o to 190), that of aldolase was 16.3 U/L (normal range, 0 to 7.6 ), and that of glycated albumin was $19.9 \%$ (normal range, 11.0 to 16.0 ). He was antinuclear antibody-positive (titer, 1:160) but negative for anti-jo-1. Computed tomography yielded no evidence of recurrent or metastatic cancer. Magnetic resonance imaging revealed signal changes compatible with inflammatory myositis in both proximal arms. Electromyography and a nerve conduction study diagnosed myopathy and peripheral sensorimotor polyneuropathy. Biopsies of the triceps brachii muscle revealed perivascular and interfascicular inflammatory CD4+ lymphocyte infil-
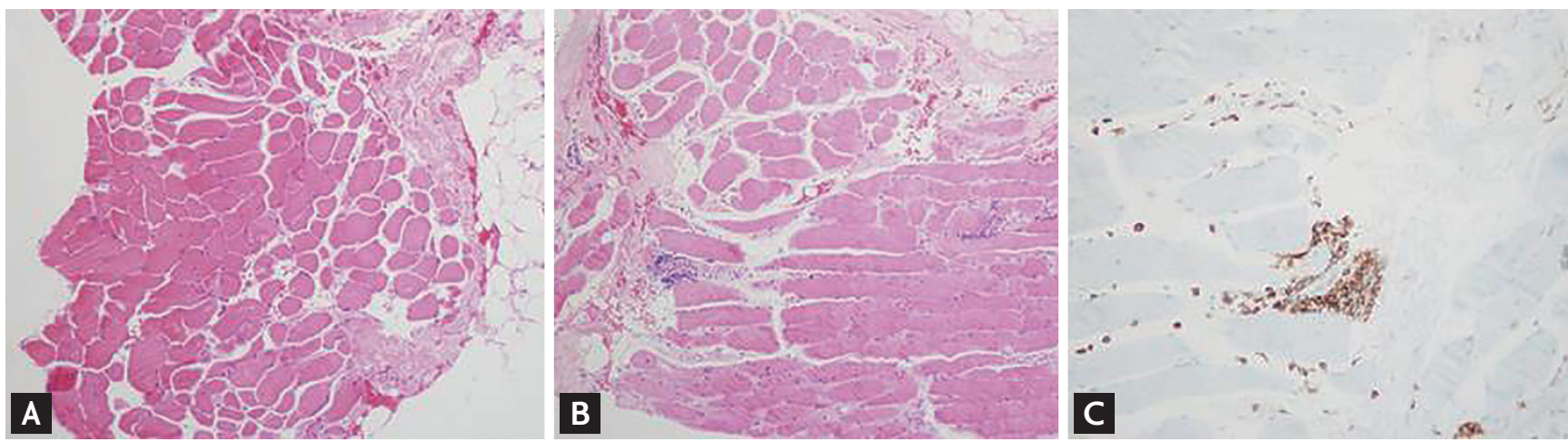

Figure 1. (A, B) Microscopically, a triceps biopsy revealed mild atrophy of perifascicular muscle fibers with occasional perimysial and perivascular inflammation (H\&E: A, ×40; B, ×100). (C) Immunohistochemically, cellular infiltrates (predominantly $\left.\mathrm{CD}_{4+}\right)$ were evident in the perimysial and endomysial regions. 

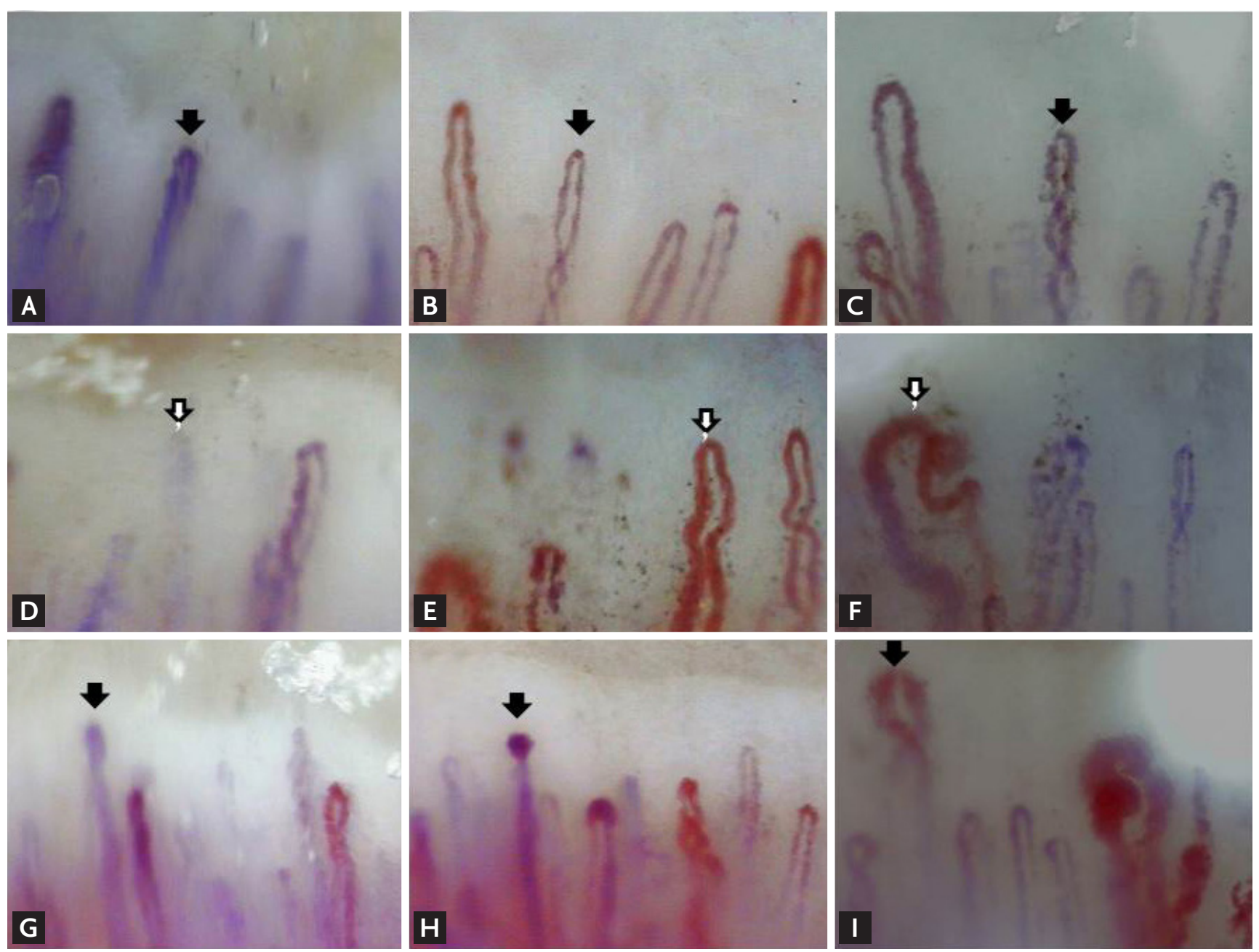

Figure 2. (A, B, C) Serial photographs of the nailbed of the third finger. (D, E, F) Serial photographs of the nailbed of the fourth finger. (G, H, I) Serial photographs of the nailbed of the right fourth finger. Arrows indicate the same capillaries. Initially (A, $\mathrm{D}, \mathrm{G})$, avascularity, moderate capillary dilation, and decreased blood flow (burred capillary margins) were apparent. After 3 months $(\mathrm{B}, \mathrm{E}, \mathrm{H})$, the avascularity was mild, the capillaries were minimally dilated, and the capillary loops were distinctly bordered. Five months later (C, F, I), the capillaries had become increasingly tortuous, megacapillaries were apparent, and skin activity had recurred.

trates with adjacent muscle fiber regeneration (Fig. 1), consistent with the histopathological findings of DM. The NFC changes included moderate avascularity, dilated capillaries, and decreased blood flow, reflecting typical DM-like microvascular involvement (Fig. 2A, $2 \mathrm{D}$, and $2 \mathrm{G}$ ). Treatment commenced with high-dose, pulsed methylprednisolone therapy (500 mg/day) for 3 days; the steroid was then tapered gradually. After 3 months, the facial rash and the painful edema of upper extremities had improved. We evaluated the NFC changes again; the mild avascularity had improved, the capillaries were only slightly dilated, and the blurred margin of the capillary loop now exhibited distinct flow (Fig. 2B, 2E, and 2H). Five months later, the facial rash and Gottron's papules recurred but the muscle enzyme levels remained normal. With recurrence of the rash, the capillaries also changed, becoming increasingly tortuous; megacapillaries were also evident (Fig. $2 \mathrm{C}, 2 \mathrm{~F}$, and $2 \mathrm{I})$.

NFC abnormalities are common in the early stages of DM. The capillary morphology is similar to that of systemic sclerosis; hence, the term scleroderma pattern, but the changes associated with systemic sclerosis develop slowly (i.e., over a relatively long period of time). Several clinical studies have explored the association between NFC changes and DM disease activity and 
clinical and laboratory findings. In a Japanese study of DM patients, capillary loss was significantly associated with muscle weakness and increased disease activity. The microhemorrhage score was associated with the severity of skin disease. Two studies found that two juvenile DM patients with normal end-row loops exhibited lower levels of skin disease activity. To date, few tests allowing evaluation of DM disease activity have been available. Our images revealed rapid characteristic changes in NFC morphology with advancing skin involvement in a DM patient. Therefore, monitoring of
NFC changes may be useful when evaluating clinical activity or the treatment response.

Written informed consents were obtained.

\section{Conflict of interest}

No potential conflict of interest relevant to this article was reported.

\section{Acknowledgments}

This study is supported by fund of the Soonchunhyang University. 